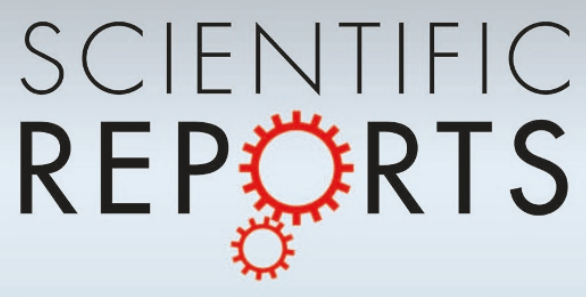

OPEN

SUBJECT AREAS:

BIOMEDICAL

ENGINEERING

ELECTRICAL AND ELECTRONIC ENGINEERING

Received

13 December 2013

Accepted

7 May 2014

Published

27 May 2014

Correspondence and requests for materials should be addressed to

W.H.L. (weihuali@ vow.edu.au)

\section{On-chip high-throughput manipulation of particles in a dielectrophoresis-active hydrophoretic focuser}

\author{
Sheng Yan ', Jun Zhang ${ }^{1}$, Ming Li' ${ }^{1}$, Gursel Alici ', Haiping Du², Ronald Sluyter ${ }^{3}$ \& Weihua Li ${ }^{1}$
}

\begin{abstract}
'School of Mechanical, Materials and Mechatronic Engineering, University of Wollongong, Wollongong, NSW 2522, Australia,
${ }^{2}$ School of Electric, Computer and Telecommunication Engineering, University of Wollongong, Wollongong, NSW 2522, Australia,

${ }^{3}$ School of Biological Sciences, University of Wollongong, Wollongong, NSW 2522, Australia.
\end{abstract}

This paper proposes a novel concept of dielectrophoresis (DEP)-active hydrophoretic focusing of micro-particles and murine erythroleukemia (MEL) cells. The DEP-active hydrophoretic platform consists of crescent shaped grooves and interdigitated electrodes that generate lateral pressure gradients. These embedded electrodes exert a negative DEP force onto the particles by pushing them into a narrow space in the channel where the particle to groove interaction is intensive and hydrophoretic ordering occurs. Particles passing through the microfluidic device are directed towards the sidewalls of the channel. The critical limitation of DEP operating at a low flow rate and the specific hydrophoretic device for focusing particles of given sizes were overcome with the proposed microfluidic device. The focusing pattern can be modulated by varying the voltage. High throughput was achieved (maximum flow rate $\sim 150 \mu \mathrm{L} \mathrm{min}{ }^{-1}$ ) with good focusing performance. The non-spherical MEL cells were utilised to verify the effectiveness of the DEP-active hydrophoretic device.

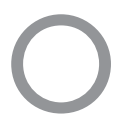

ver the past few decades microfluidic platforms have made significant progress due to their reduced consumption of reagents, fast assay preparation, portability, and ease of integration with multiple sample processing ${ }^{1}$. Manipulating particles in microfluidic devices has a variety of applications, such as trapping, focusing, and sorting cells and micro-particles. One application of focusing particles into a narrow stream to enhance the performance of flow cytometry which is then utilised for counting, detecting, and sorting microbeads ${ }^{2}$ has already been developed; this application can be categorised into the active and passive manipulation of particles. Active manipulation utilises external energy such as acoustophoresis ${ }^{3}$, magnetophoresis ${ }^{4}$ and dielectrophoresis(DEP) ${ }^{5-16}$, whereas passive manipulation includes hydrodynamic ${ }^{17-20}$, inertial ${ }^{21}$, and hydrophoretic ${ }^{22-26}$ methodologies that can be used to dominate the trajectory of particles. In general, active techniques are precise methods to control the target particles. DEP has made significant progress over the last decade due to its label-free nature. Dielectrophoretic particle focusing is primarily based on the non-uniform AC electric field induced by microelectrodes inside the channels $s^{6-9}$ where the particles are pumped by a pressurised flow and then focused to a tight stream by balancing forces ${ }^{27}$. Despite its advantages, increasing the throughput of DEP-based devices when focusing particles remains an ongoing research problem. Some researchers have made progress in improving the flow rate of DEP-based devices. Markx et $a^{28}$ used AC DEP to separate viable and non-viable yeast cells at a flow rate of $500 \mu \mathrm{L} \mathrm{min}^{-1}$. Huang et al $^{29}$ introduced DEP forces for field-flow fractionation, and high flow rate of $160 \mu \mathrm{L} \mathrm{min}^{-1}$ was achieved in a $200-\mu \mathrm{m}$ high by $17-\mathrm{mm}$ wide micro-channel. Park et al. ${ }^{1}$ proposed a novel microfluidic platform that combined a separation channel and a concentration channel into a single device. E.coli was focused and separated from human cerebrospinal fluid. 94.3\% of E.coli was separated and the flow rates of sheath flow and sample stream were set at $5 \mu \mathrm{L} \mathrm{min}^{-1}$ and $0.5 \mu \mathrm{L} \mathrm{min}{ }^{-1}$, respectively. Cheng et al. ${ }^{30}$ reported a high-throughput continuous particle sorter utilising a 3D travelling-wave DEP. Red blood cells were effectively focused and separated from heterogeneous samples that were filled with debris, with a maximum flow rate of $10 \mu \mathrm{L} \mathrm{min}{ }^{-1}$. Although direct current (DC) DEP can focus particles ${ }^{16}$, high voltages are needed to generate enough DEP force, which may then generate Joule heating and lead to the formation of bubbles. Additionally, DC DEP is a non-pumping method for particle focusing where the particles are driven by electro-osmotic flow ${ }^{31}$ and migrate at a relatively low speed. Hydrodynamic focusing based on a passive method is still simple and effective, although focusing depends mainly on the accuracy of flow control, because any unstable pumping will lead to an imperfect confinement of particles and deflect them from their initial positions. Huang et al. ${ }^{32}$ proposed a 
deterministic lateral displacement (DLD) to continuously separate beads with high resolution which demonstrated, successfully separated blood cells and isolated plasma from whole blood ${ }^{33}$. DLD can be combined with DEP to improve the separation efficiency. Beech et $a l^{34}$ utilised DEP force and steric force to move particles having a diameter close to the critical diameter in the displacement mode. But fabricating an array of posts in a micro-channel may prove to be challenging. A novel approach to focus particles based on hydrophoresis has recently been reported ${ }^{24}$.

Hydrophoresis is a sheathless and passive method that utilises a pressure gradient induced by the microstructure and steric effect between the particles and grooves ${ }^{26}$. The concept of hydrophoresis has demonstrated its capability by separating micro-particles and isolating white blood cells (WBCs) from $\mathrm{RBCs}^{24,35}$. This new technique has a promising future in manipulating particles in a microchannel because of the ease of parallelisation. A hybrid method of cell rolling and hydrophoresis has been proposed by using a parallelised device consisting of 20 channels $^{36}$. Hydrophoretic ordering has been studied experimentally because the particles can be ordered and focussed by hydrophoresis and therefore its diameter should normally be larger than half the height of the channel ${ }^{23}$. However, it does have some limitations because the particles being processed in the hydrophoretic experiment were around $10 \mu \mathrm{m}$ in diameter, which means the height of the channel was usually less than $20 \mu \mathrm{m}$, and the channels were $50 \mu \mathrm{m}$ wide, which made it difficult to achieve high throughput. As for DNA separation ${ }^{23}$, the cost of fabricating a $1.2 \mu \mathrm{m}$ high channel is very high because of the accurate resolution and high precision needed for photolithography. Meanwhile, narrow channels may lead to higher flow resistance, which means that more energy will be consumed by the syringe pump, and the channels can easily be blocked by dust from the air. Even though hydrophoretic filtration has a high resolution where $11 \mu \mathrm{m}$ and $12 \mu \mathrm{m}$ particles can be separated in a micro-channel with slanted obstacles and filtration obstacles $^{35}$, hydrophoretic devices still lack flexibility. Once a channel is fabricated, its height is fixed at a certain value so the size of particles which can be focused and separated is determined in advance by the height of the channel.

In this paper we first propose the concept of "DEP-active hydrophoretic focusing" which is a combination of DEP and hydrophoresis. The critical limitation of DEP operating at a low flow rate and the specific hydrophoretic device for focusing particles with a given size are overcome in our microfluidic device. Here the particle is levitated by negative AC-DEP and its equilibrium position in a micro-channel relies on the magnitude of the electric field. The stronger the electric field is, the higher the equilibrium position the particles have. This means that interaction between the particle and groove becomes intensive and steric hindrance occurs even though hydrophoretic ordering has not been satisfied and the diameter of the particle is less than half the height of the channel (Fig. 1a). Whether the particles can be focused or not is determined by the external electric field. A particle with a diameter that is less than half the height of the channel can still be focused in our channel without redesigning and fabricating a new channel. In this study the anisotropic flow resistance is induced by crescent-shaped grooves to focus the micro-particles and murine erythroleukemia (MEL) cells into sidewalls of the crescent-shaped micro-channel (CSM). Compared to traditional hydrophoretic devices, CSM has a larger cross section which makes it easy to achieve high throughput. The effects of applied voltage, flow rate, and particle diameter on DEP-active hydrophoretic focusing were investigated and DEPactive hydrophoretic focusing of MEL cells in a CSM has been demonstrated.

\section{Results}

The motion of particles in a DEP-active hydrophoretic device. In a non-uniform electric field the polarisable particles suspended in an
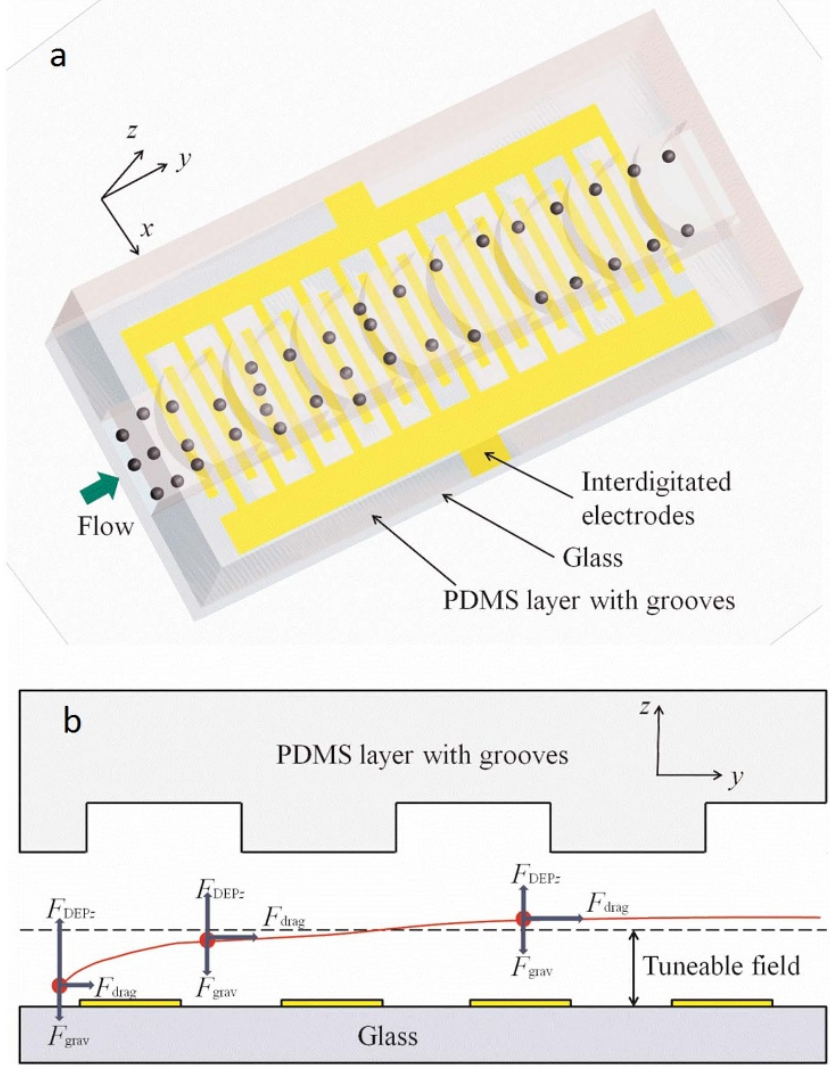

Figure 1 DEP-active hydrophoretic focusing. (a) Overview of device showing the crescent-shaped channel on top of the interdigitated electrodes. Particles, levitated by negative DEP force in z-direction, will interact with grooves along which a pressure gradient is induced by the anisotropy of flow resistance and reach each side of channel. (b)

Schematics of the particle trajectory in cross-section along y-direction. A giving particle has tunable equilibrium height under different electric field.

aquatic medium will experience a dieletrophoretic force. The net force $F_{\mathrm{DEP}}$, are given $\mathrm{by}^{37}$ :

$$
F_{\mathrm{DEP}}=2 \pi \varepsilon_{m} r^{2} \operatorname{Re}[K(\omega)] \nabla E_{\mathrm{RMS}}{ }^{2}
$$

where $\varepsilon_{m}$ is the absolute permittivity of the suspending medium, and $r$ denotes the particle radius, $E_{\mathrm{RMS}}$ is the root-mean-square value of the applied electric field, $\nabla E_{\mathrm{RMS}}{ }^{2}$ is the gradient of the square of the applied field $E_{\mathrm{RMS}}$, Re indicates the real part. $K(\omega)$ refers to the Clausius-Mossotti (CM) factor which depends on the complex permittivities of the particle and the suspending medium, and the frequency of the external electric field as well:

$$
K(\omega)=\frac{\varepsilon_{p}^{*}-\varepsilon_{m}^{*}}{\varepsilon_{p}^{*}+2 \varepsilon_{m}^{*}}
$$

where $\varepsilon^{*}=\varepsilon-i \sigma / \omega(i=\sqrt{-1})$ is the complex permittivities, $\sigma$ is the electrical conductivity, and $\omega$ is the frequency of the electric field. The subscripts $(p, m)$ represents the particle and suspending medium, respectively. The $K(\omega)$ factor, a dominating role in DEP force, represents the dielectric properties of particles and suspending medium under different frequencies of the electric field applied. If the permittivity of a particle is greater than that of the suspending medium $(K(\omega)>0)$, a positive DEP (p-DEP) is generated in this mode, where the particle migrates to the region of a strong electric field. However, if $K(\omega)<0$, the motion of the particle is repelled from the region of a strong electric field, which is termed negative DEP (n-DEP). 
In our focusing experiment, the particles experiencing an n-DEP force will be levitated in the suspending medium. Theoretical modelling of the CM factor for different particles types was reported by Park et $a l^{1}$. The DEP force also relies on the square of the applied field, which implies that the larger the electric field applied, the greater DEP force will act on the particles. The magnitude of the DEP force reduces exponentially with increasing distance above the electrodes, which drives the particles to an equilibrium position by balancing the DEP force and a sendimentation force $F_{\text {grav }}$ in the $z$ direction. The equilibrium position of a given particle is tunable by applying a different electric field (Fig. 1b).

When fluid passes through a curved channel, the fluid near the centre has a higher inertia than fluid close to the walls and tends to move outwards around a curve that in turn creates a pressure gradient induced by the anisotropy of flow resistance. Because the channel is sealed, fluid near the walls recirculates inwards due to the centrifugal pressure gradient, inducing two symmetric vortices which is called Dean flow ${ }^{21}$. In a curved channel, two dimensionless numbers that characterise the Dean flow are defined as the Dean number $D e=\operatorname{Re}(H / 2 R)^{1 / 2}$ and curvature ratio $\delta=H / 2 R$, where $R$ is the radius of the curvature of the channel and $H$ is the width of the channel. The Dean flow velocity scales as $U_{D} \sim D e^{221}$.

In the CSM (Fig. 2), due to the low resistance provided by grooves in the cross section, fluid will fill the grooves and induce a transverse movement within the channel. The pressure of the fluid in the central grooves is higher than that at the sides. This anisotropic structure generates a pressure gradient from the central grooves to the sides which induces helical recirculation ${ }^{2}$, where the helical streams rotate like a gear following a sequence of upwards, deviation, downwards, focusing, and then upwards again (the insets of Fig. 2a).

Particles suspended in the medium have helical motions that follow the flow of the fluid. Particles also experience four stages in each cycle: deviation, downwards, focusing, and upwards (Fig. 2b). This deviation in the flow brings particles to the side of the channel where the velocity is relatively low in bulk flow (the blue line in the Fig. 2b). The particles move down in downwards flow and then the focusing flow brings the particles to the centreline of the channel where the velocity in bulk flow is higher than at the walls. Meanwhile, particles will migrate along the bottom of channel and then move up following upwards flow. The cycle of focusing and upwards is longer than deviation and downwards (as shown in Fig. 2b), mainly because the particle velocity during focusing and upwards is faster than deviation and downwards. In this way the particles will move back and forth in the channel.

Hydrophoresis utilises a steric hindrance mechanism to separate or focus particles under a pressure gradient induced by anisotropic flow resistance ${ }^{23}$. The steric effect arises when atoms in a molecule are brought too close together. Atoms are likely to occupy preferred positions rather than overlap each other, a principal that works well in micro-scale physics. As a giving particle ( $D$ in diameter) reaches the proximity of the sidewall, then Dean flow brings it to the central area of the channel and pushes it upward, driving it to align with the surface of the groove (as shown in Fig. $2 \mathrm{~b}$ and Fig. 3a left). When a particle is comparable in size with the height of the channel $\left(H_{g}\right)$, steric hindrance prevents a particle following the Dean flow. Thus, particles keep moving near the sidewall instead of migrating back and forth in the channel. This hydrophoretic ordering is largely determined by the height of the channel. Where $D / H_{g} \geq 0.5$, the grooves begin to hinder particles following the Dean flow induced by anisotropic microstructures and causes hydrophoretic ordering ${ }^{23}$.

In short, interaction between particle and groove deflects those particles that are almost the same size as the height of channel from their normal trajectories, and leads to a new equivalent flow path which is called hydrophoretic ordering. Those particles experiencing a negative DEP force will be levitated in the suspending medium in the presence of the electric field, which leads to more interaction

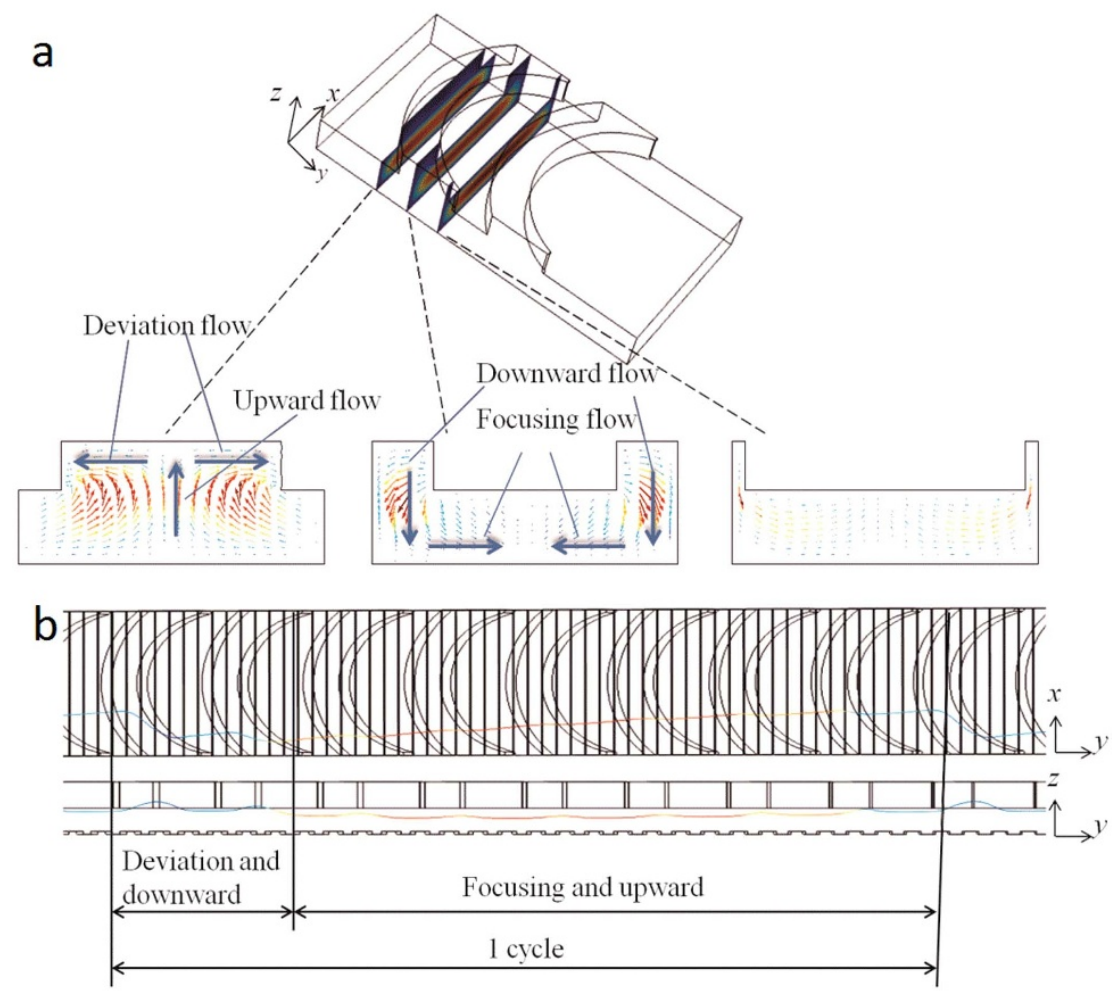

Figure $2 \mid$ Dean flow. (a) The schematic diagrams of the CSM and the simulated results of flow field in the cross-sections. The flow direction is along the yaxis. The anisotropic microstructure induces helical recirculation, including an upwards, deviation, downwards, and focusing flow. (b) Particle trajectory in top view and side view. The particles follow the flow field and experience four stages in each cycle: deviation, downward, focusing, and upward. Blue represents low particle velocity, while red refers to high particle velocity. 

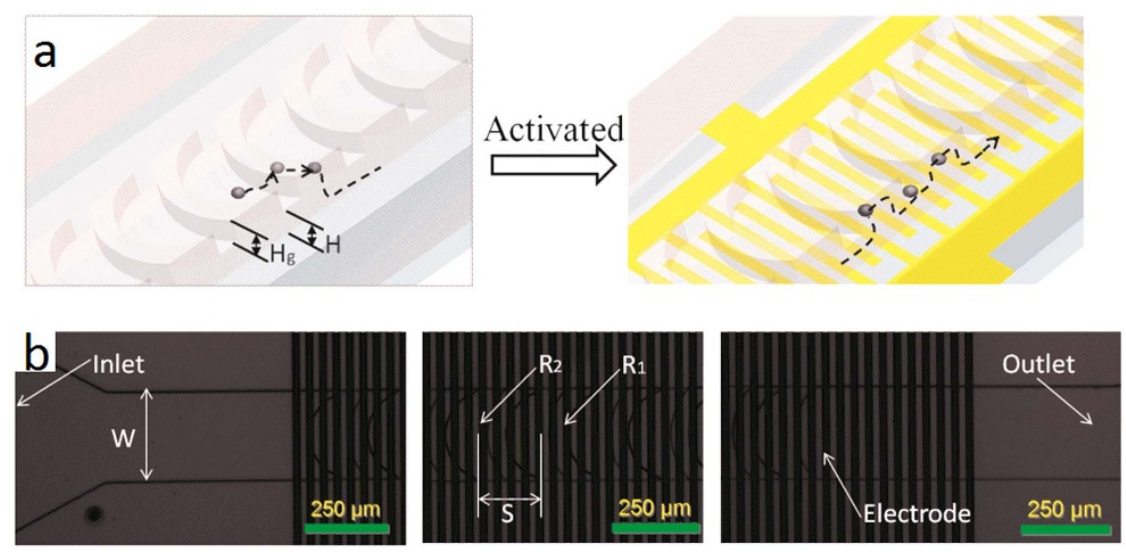

Figure $3 \mid$ Microfluidic device for DEP-active hydrophoretic particle focusing. (a) Particles not obeying the hydrophoretic ordering migrate back and forth in the channel (Left), while they can be focused to the sidewalls of channel in the presence of an external electric field at the same conditions(Right). (b) Optical micrographs of the crescent-shaped grooves in the focusing channel with specific geometric parameters of R $1=125 \mu \mathrm{m}$, $\mathrm{R} 2=175 \mu \mathrm{m}, \mathrm{W}=250 \mu \mathrm{m}, \mathrm{H}=45 \mu \mathrm{m}, \mathrm{Hg}=45 \mu \mathrm{m}$, and $\mathrm{S}=175 \mu \mathrm{m}$. The crescent-shaped grooves were formed on the top of the channel.

between particles and grooves. Therefore, even though the diameter of the particles is less than half of the channel they still have some possibility for hydrophoretic ordering when they migrate along the equivalent flow paths and remain close to the sidewalls of the channels without deviation after the electrodes have been activated (Fig. 3a). The height that particles are levitated relies on the magnitude of the electric field. Assuming that the particles are pushed high enough to lead to hydrophoretic ordering, they will focus on the sidewalls of the channel, but larger particles, exerting a greater DEP force, will be levitated to a higher position in the $z$-direction, which means the interaction between particles and grooves is more intensive and hydrophoretic ordering will arise easily. Therefore, whether the particles can be focused on each side of channel or not is tunable.

DEP-active hydrophoretic focusing. We first tested the effect of applied voltage on DEP-active hydrophoretic focusing. Using the device with crescent-shaped grooves and interdigitated electrodes, we observed that beads passing through them showed different patterns. In this experiment $10 \mu \mathrm{m}$ diameter particles were introduced and the flow rate was $20 \mu \mathrm{L} \mathrm{min}{ }^{-1}$. The trajectories of the particles in each image were captured at a time interval of $1 / 7 \mathrm{~s}$ and the images were superposed. The particles were smaller than half of the height of the channel in diameter, which did not satisfy hydrophoretic ordering, so they migrated back and forth inside the channel (as shown in Fig. 2b) and could not form hydrophoretic focusing (the right of Fig. 4a) without an electric field. However, the particles did tend to focus on the sidewalls of the channel when a voltage of $5 \mathrm{~V}_{\mathrm{p}-\mathrm{p}}$ at a frequency of $1 \mathrm{MHz}$ (the right of Fig. 4c) was applied. At this frequency beads were subject to a negative DEP force and were levitated to a higher position in the channel which resulted in a more intensive steric interaction between particle and groove that led to hydrophoretic ordering. Regardless of their initial positions, beads were focused onto each side of the channel. As Fig. $4 \mathrm{e}$ shows, the focused positions were closer to the sidewalls of the channel as the voltages increased, such that the higher the voltages applied, the less focused were the widths.

Next, we examined the effect of the flow rate on DEP-active hydrophoretic focusing. The influence of the flow rate on the focusing position and width of the particle was investigated in the CSM with voltages of $5 \mathrm{~V}_{\mathrm{p} \text {-p }}$ applied and a flow rate ranging from 10 to $200 \mu \mathrm{L}$ $\mathrm{min}^{-1}$. Particles of $13 \mu \mathrm{m}$ were introduced into the CSM. The crescent-shaped grooves in a channel were omitted, and the linear velocities of particles flowing through the $250 \mu \mathrm{m}$ wide by $45 \mu \mathrm{m}$ high channel ranged from 1.48 to $29.63 \mathrm{~cm} \mathrm{~s}^{-1}$. Figure 5 showed that the beads were focused on each sidewall of the channel and had two separated peaks from $51.19 \pm 7.76 \mu \mathrm{m}$ and $190.79 \pm 7.76 \mu \mathrm{m}$ to
$43.43 \pm 6.20 \mu \mathrm{m}$ and $196.28 \pm 6.20 \mu \mathrm{m}$, and a flow rate that changed from 10 to $150 \mu \mathrm{L} \mathrm{min}{ }^{-1}$. Although the flow rate was up to $150 \mu \mathrm{L}$ $\min ^{-1}$, the particles were still focused well into the channel.

We then investigated the effect of particle diameter on DEP-active hydrophoretic focusing. Particles of 8-, 10-, and 13- $\mu \mathrm{m}$ diameter were introduced into the micro-channel at a flow rate of $20 \mu \mathrm{L}$ $\min ^{-1}$. Figure $6 \mathrm{a}$ shows the focusing profiles with different particle diameters and without an external electric field. The particles were evenly distributed at the outlet as they were injected from the inlet. Although the 13- $\mu \mathrm{m}$ particles had a tendency to focus onto the sides of channel, some particles moved towards the centre of the channel. Those particles with different diameters had clear focusing profiles at an applied voltage of $8 \mathrm{~V}_{\mathrm{p} \text {-p }}$ (Fig. 6b), and the separated peaks were symmetrical about the centreline of the channel. As we increased the voltage from 0 to $8 \mathrm{~V}_{\mathrm{p} \text {-p }}$, the focusing widths of particles with different diameters decreased rapidly (Fig. 6c). The focusing widths fell from 86.65 to $23.81 \mu \mathrm{m}$ for 8 - $\mu \mathrm{m}$ beads. We observed a similar trend with the $10-\mu \mathrm{m}$ beads, where the focusing widths were $82.46,38.72$, 20.40 , and $15.30 \mu \mathrm{m}$ for $0,2,5$, and $8 \mathrm{~V}_{\mathrm{p} \text {-p }}$, respectively. With the 13 $\mu \mathrm{m}$ beads, the focusing width decreased to $17.05 \mu \mathrm{m}$ at a voltage of 2 $\mathrm{V}_{\mathrm{p}-\mathrm{p}}$. Then the focusing width reduced to $13.94 \mu \mathrm{m}$ when the voltage applied went to $8 \mathrm{~V}_{\mathrm{p} \text {-p }}$.

Most cells in nature are not completely spherical and exhibit distributions in cell size. To verify whether non-spherical particles can be focused in our DEP-active hydrophoretic device, MEL cells (12 $\mu \mathrm{m}$ in diameter) were used in our experiment. The cells passed quickly into the channel, which proved difficult to capture with the CCD camera, so a flow rate of $1 \mu \mathrm{L} \mathrm{min}{ }^{-1}$ was selected. In this condition the trajectories of the cells were recorded in micrographs with stretched lines, as shown in Figure 7a. The inset describes the morphology of MEL cells. The lateral positions of the cells were measured, and then the number of particles that appeared in different positions was counted manually (Fig. $7 \mathrm{~b}$ ). After passing the microfluidic device, approximately $44.2 \%$ and $50.7 \%$ of the MEL cells went through the channel within the lateral range from 25 to $75 \mu \mathrm{m}$ and from 175 to $225 \mu \mathrm{m}$.

\section{Discussion}

In order to better understand these DEP-active hydrophoretic phenomena, a numerical simulation was conducted utilising COMSOL software and the particle trajectories and electric fields were calculated. The particles utilised for this simulation were $10 \mu \mathrm{m}$ in diameter. A fluid flow was applied along the $y$-axis at a flow rate of $20 \mu \mathrm{L}$ $\min ^{-1}$. The particle trajectories were simulated without an electric field and with an electric field in Fig. 5a. This software has some 
Inlet

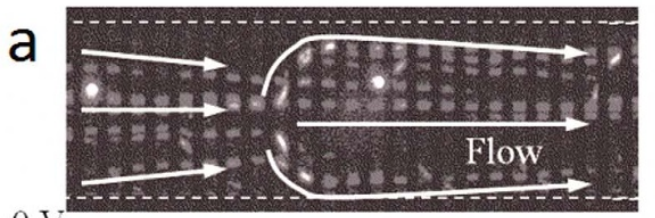

$0 \mathrm{~V}$

b

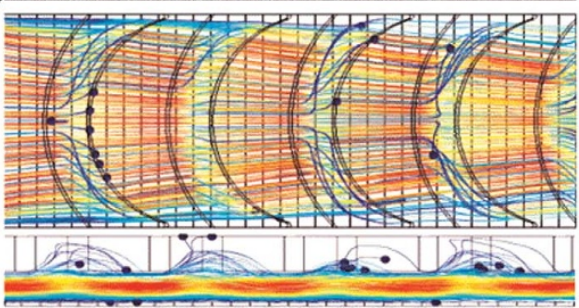

C

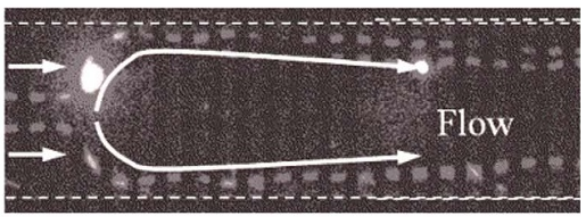

$5 \mathrm{~V}$
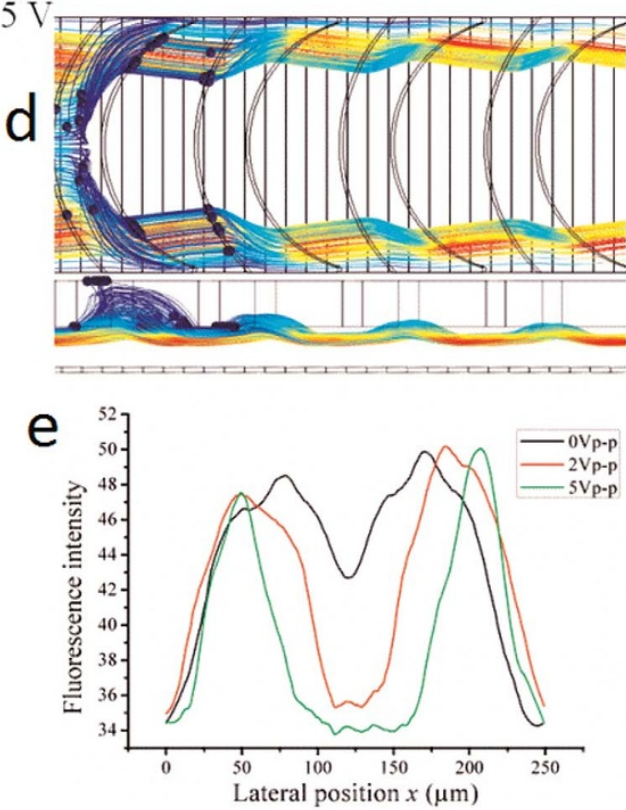

Outlet
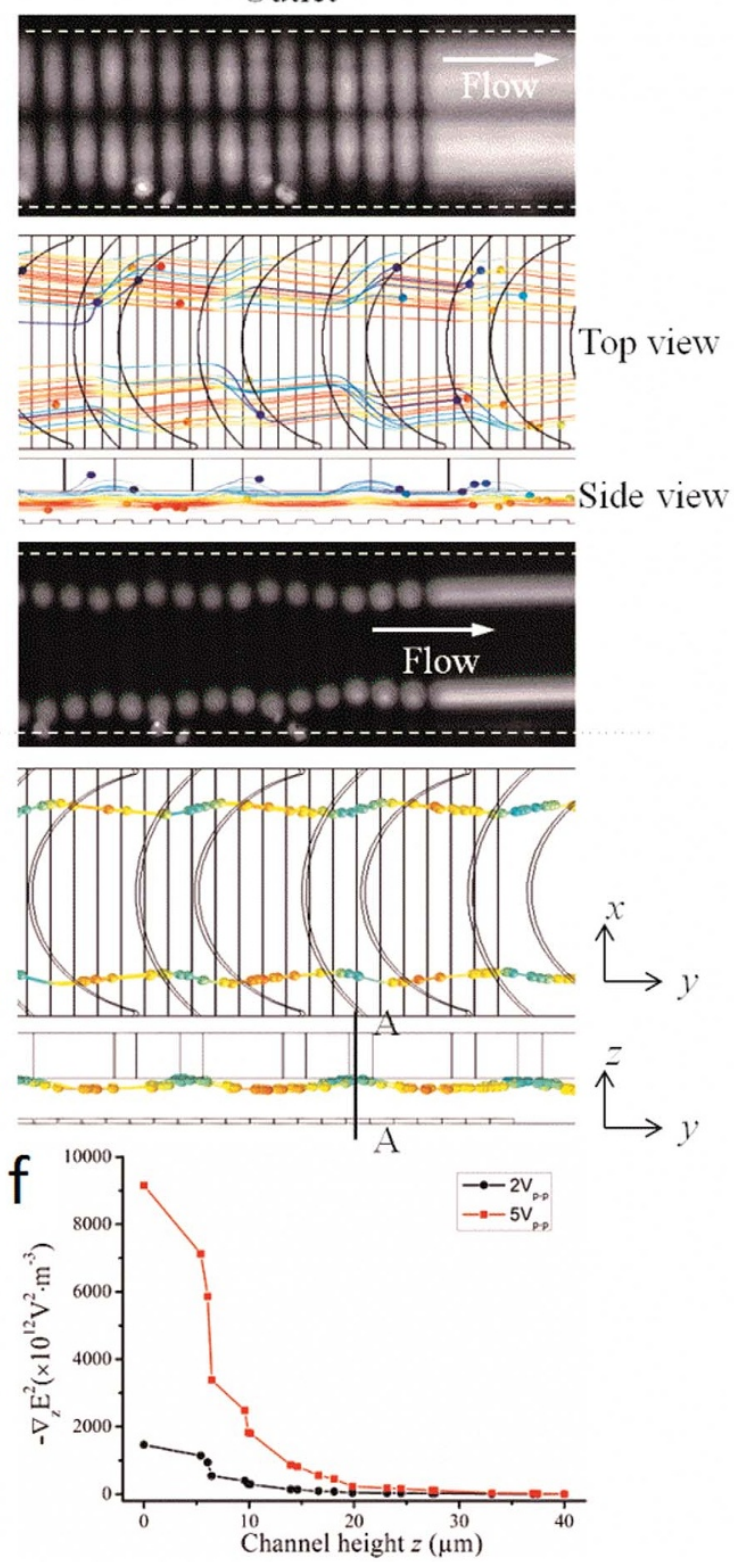

Figure $4 \mid$ Experimentally focused patterns and numerically predicted particle trajectories of $10-\mu \mathrm{m}$ particles at various voltages. The applied flow rate was $20 \mu \mathrm{L} \mathrm{min}{ }^{-1}$. The beads were evenly distributed at the inlet. The simulation geometry had the same geometric dimensions with the experimental channel. Optical micrographs showing the voltage-dependent focusing positions of (a) $0 \mathrm{Vp}$-p and (c) $5 \mathrm{Vp}$-p. (b) Particle trajectory at both the inlet (Left) and the outlet (Right) without an external voltage. Particles migrated back and forth and were not focused in the channel. (d) Particle trajectory at both the inlet (Left) and the outlet (Right) at voltages of $5 \mathrm{Vp}$-p. Particles focused onto the sidewalls of the channel with the effect of steric hindrance. (e) Measured focusing profiles under different applied voltages. (f) Plot of the calculated gradients of the square of the applied field in the $\mathrm{z}$-direction along the line, A-A.

drawbacks; the particles were immobilised when they touched the groove, which is a mismatch to the actual situation, and we also plotted the calculated gradients of the square of the applied field in $z$-direction along the line A-A in Fig. $4 \mathrm{~d}$ to investigate the electric fields at various voltages.

Without an external electric field, particles that were released evenly into the inlet occupied the whole channel in the $z$-direction (the left of Fig. 4b), but after the electrodes were excited, the particles were pushed into a narrow space in the channel where the steric interaction between the particles and grooves occurred (Fig. 4d). In this way the $10 \mu \mathrm{m}$-diameter microspheres remained in their focused position, which perfectly matched the experimental results (Fig. 4c). Figure $4 \mathrm{f}$ shows the simulated gradients of the square of the field applied in the $z$-axis at 2 and $5 \mathrm{~V}_{\mathrm{p}-\mathrm{p}}$ voltages where the maximum intensity of the electric field gradient at $5 \mathrm{~V}_{\mathrm{p}-\mathrm{p}}$ was $\sim 5$ times as high as that at $2 \mathrm{~V}_{\mathrm{p}-\mathrm{p}}$. The gradients of the electric field reduced exponentially as the distance above the electrodes increased, which is consistent with the conclusion from Pethig et al. ${ }^{38}$. Compared with a voltage of $2 \mathrm{~V}_{\mathrm{p}-\mathrm{p}}$, the particles located at the lower position ( $\sim 20 \mu \mathrm{m}$ or less) exerted a larger DEP force and pushed quickly to higher positions when a voltage of $5 \mathrm{~V}_{\mathrm{p} \text {-p }}$ was applied. Thus, the focusing performance at $5 \mathrm{~V}_{\mathrm{p}-\mathrm{p}}$ was better than that at $2 \mathrm{~V}_{\mathrm{p}-\mathrm{p}}$.

As Fig. 5 shows, the increasing flow rate had little effect on the focused position, a result that was consistent with the previous work of Park and $\mathrm{Choi}^{24}$, who reported that the particle position was independent of the flow rate when the flow rate range was less than 


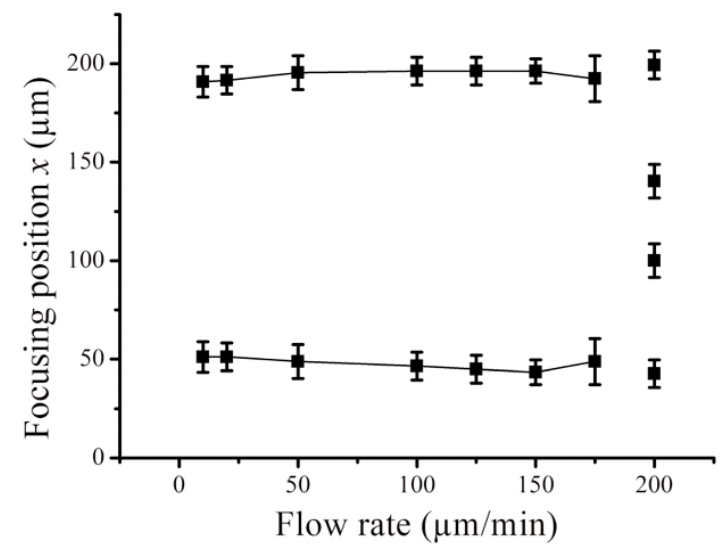

Figure 5 Focused positions and widths of the $13 \mu \mathrm{m}$ particles. The flow rate was changing from 10 to $200 \mu \mathrm{L} \mathrm{min}^{-1}$. The applied voltage on the electrodes was $5 \mathrm{Vp}$-p. Each focusing position and width was obtained from measuring the fluorescent profile.

$5 \mu \mathrm{L} \mathrm{min}{ }^{-1}$. The maximum flow rate for focusing with good performance in our experiment was $\sim 30$ times as high as that reported by Park and $\mathrm{Choi}^{24}$. The pressure gradient between the centre and sidewall of the channel increased with the increasing flow rate and forced the particles to move slightly closer to the sidewall of the channel. However, after the flow rate increased to $175 \mu \mathrm{L} \mathrm{min}{ }^{-1}$ the particles focussed very well and the focusing position moved to the centreline of the channel and increased in width. When the flow rate went to $200 \mu \mathrm{L} \mathrm{min}^{-1}$ the particles did not focus any more. Since the particles passed through the channel very quickly, they were exposed to the electric field for a shorter time and could not be levitated high enough to generate a hydrophoretic ordering so the focusing performance deteriorated.

The influence of particle diameter on the focusing width was strong because the larger particles experiencing a higher DEP force had a higher equilibrium position by balancing the DEP force and gravity in the $z$-axis. Therefore, larger particles were pushed into a narrower space where hydrophoretic ordering occurred easily and the particles were focused onto the sidewalls of the channel. Because the magnitude of the electric field gradients reduced exponentially as the distance above the bottom of the channel increased (Fig. 4f), they remained almost the same when the height was up to $20 \mu \mathrm{m}$ or more, and as the applied voltage increased. Once the particles levitated to $20 \mu \mathrm{m}$ or higher, where the DEP force was less dominant, the focusing performance changed slightly with an increase in the external electric field (Fig. 6c). Figure 7b showed the focused positions of MEL cells were symmetrical about the centre of the channel, which showed a similar focusing movement with the beads. Even though the diameter of the MEL cells was less than half of the channel height, which did not satisfy the condition required for hydrophoretic ordering, they were still focused on the sidewalls of channel at $5 \mathrm{~V}_{\mathrm{p}-\mathrm{p}}$. Therefore, the DEP-active hydrophoretic device can be utilised for biological cells and it will provide a tunable microfluidic system for clinical diagnostics.

\footnotetext{
Methods

Design and fabrication of microfluidic device. Details of the fabrication were reported in our previous work ${ }^{39,40}$. Briefly, an electrode layer of $50 \mathrm{~nm} \mathrm{Ti} / 150 \mathrm{~nm} \mathrm{Pt}$ was then patterned with a standard lift-off process. The spacing and width of the interdigitated electrodes were both $20 \mu \mathrm{m}$. The SU-8 master mould, patterned on a silicon wafer, was fabricated by two-step photolithography. The channel consisted of 60 units of identical crescent-shaped grooves, each of which had a small and large curvature of $125 \mu \mathrm{m}\left(R_{1}\right)$ and $175 \mu \mathrm{m}\left(R_{2}\right)$, respectively (Fig. 3b). The width of the channel $W$ was $250 \mu \mathrm{m}$, the height was $H=45 \mu \mathrm{m}$, the height of the grooves was $H_{g}=45 \mu \mathrm{m}$, and the pitch between the grooves was $S=175 \mu \mathrm{m}$.
}
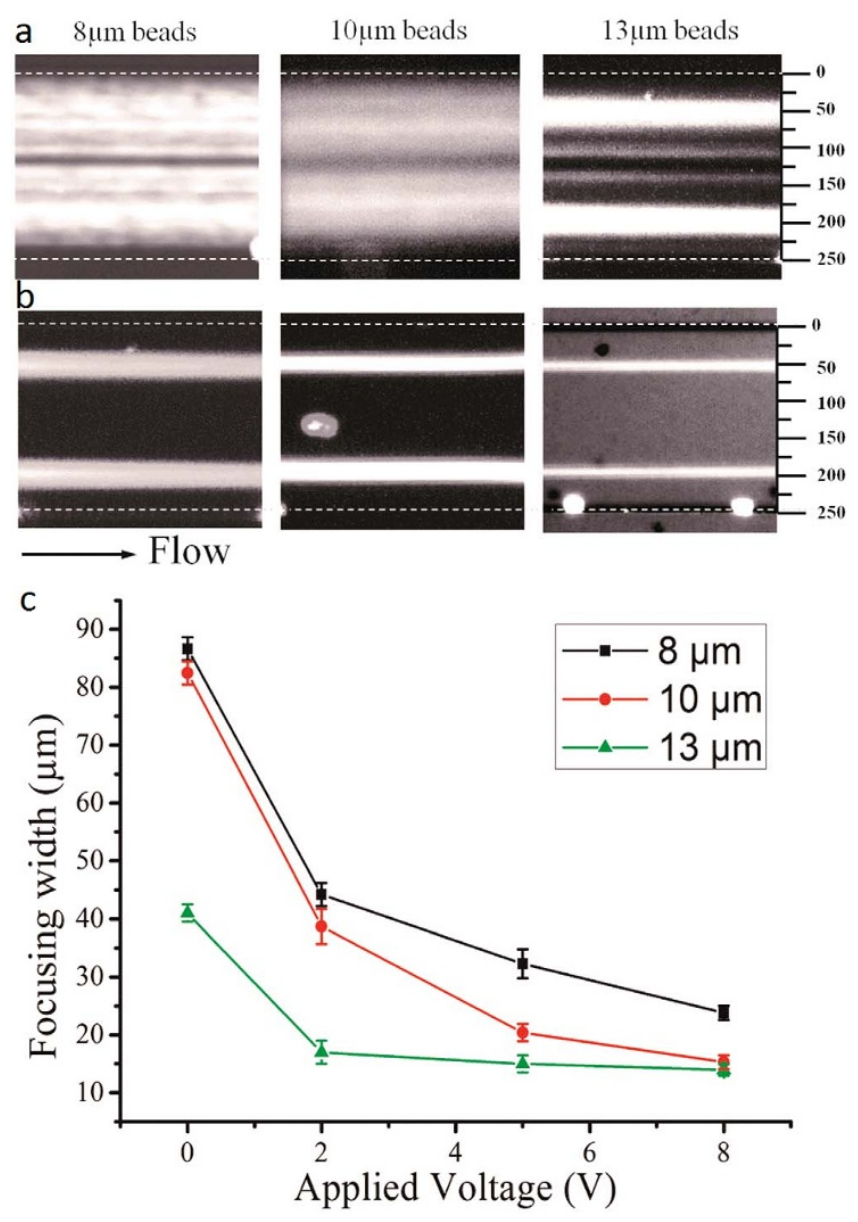

Figure $6 \mid$ Focusing patterns of 8-, 10-, and 13- $\mu \mathrm{m}$ particles. The applied voltage was changing from 0 to $8 \mathrm{~V}_{\mathrm{p} \text {-p }}$. The applied flow rate was $20 \mu \mathrm{L}$ $\mathrm{min}^{-1}$. The particles had a uniform distribution at the inlet. (a) Optical microscopy images showing the size-dependent focusing patterns in the absence of applied voltage and (b) at an applied voltage of $8 \mathrm{~V}_{\mathrm{p}-\mathrm{p}}$. (c) Measured focused widths from the fluorescent profiles. Average value of 3 times of measurement.

CV18\%), $10 \mu \mathrm{m}$ (Product No. G1000, CV5\%), and $13 \mu \mathrm{m}$ (Product No. 36-4, $\mathrm{CV} 16 \%)$ in diameter were used in our experiments. The final concentration was about $10^{6}$ beads per millilitre after diluting them with deionized water.

MEL cells were kindly supplied by Dr. Sally A. Eaton (University of Sydney, Sydney, Australia). The cell lines were maintained in complete culture medium (RPMI-1640 medium containing $10 \%$ fetal serum and $5 \mathrm{mM} \mathrm{L}$-glutamine) at $37^{\circ} \mathrm{C}$ and $95 \%$ air $/ 5 \% \mathrm{CO}_{2}$. MEL cells were marked using PKH26 red fluorescent cell linker kit (SIGMA-ALDRICH, Product No. P9691). The detailed procedure refers to technical guidelines provided by SIGMA-ALDRICH.

Experimental setup. The AC electric field was generated by a function generator (33250A, Agilent, USA). The copper wires soldered onto the platinum pads were connected to the function generator to activate the electrodes. Prepared particle suspension was pumped into the channel with a syringe pump (Legato 100, Kd Scientific). The movement of particles through the CSM was monitored by an inverted microscope (CKX41, Olympus, Japan) and captured by a CCD camera (Rolera Bolt, Q-imaging, Australia). The images were post-processed by the software Q-Capture Pro 7 (Q-imaging, Australia), and images of particle tracing were acquired by overlaying the consecutive images. The fluorescent profiles were taken from the outlet to measure the focusing performance of this device. The focusing width was defined as the distance between two points where the intensity value occupied half of the threshold. The focusing position was measured from the middle of these two points. As for DEP-active hydrophoretic focusing of MEL cells, cells flowing through the CSM were recorded in consecutive images ( 400 frames; 7 frames per second). The cells passing through different positions in the channel were counted manually. Focusing experiments were repeated three times. Electrodes were excited with a 1$\mathrm{MHz}$ sine wave for microspheres and with a $10-\mathrm{MHz}$ sine wave for MEL cells.

Numerical simulation. COMSOL Multi-physics 4.3 (COMSOL, Burlington, MA) finite element software was used to calculate the flow field and electric field in CSM. A 

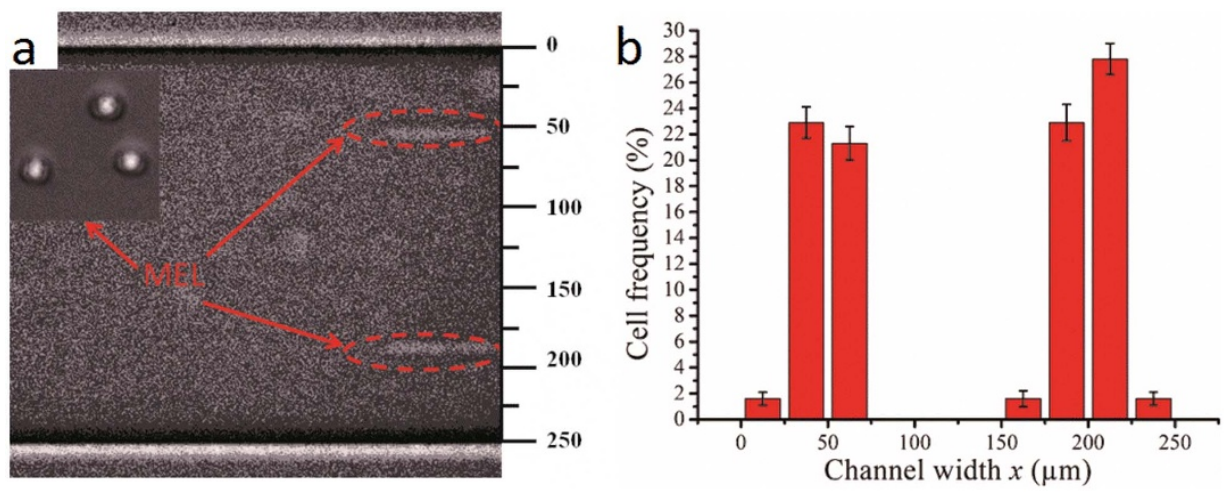

Figure 7 Focusing patterns of MEL cells. The applied flow rate was $1 \mu \mathrm{L} \min ^{-1}$ and applied voltage was $5 \mathrm{~V}_{\mathrm{p} \text {-p }}$ at a frequency of $10 \mathrm{MHz}$. The cells were evenly distributed at the inlet. (a) Optical microscopy images showing the focusing patterns of the cells in the microfluidic device. The inset describes the morphology of the cells. (b) Cell frequency at different lateral positions. Average value of 3 times of measurement.

laminar steady incompressible flow model was used because the maximum Reynolds number in the experiments was about 30 , which is much less than the 2300 that is normally considered as the transition number from laminar to turbulent flow ${ }^{41}$. An electric current module was applied to calculate the distribution of electric field in the channel, which will create the DEP force on particles. Another module, particle tracing for fluid flow, can couple laminar flow module and electric field together to simulate particle trajectory in the micro-channel.

1. Park, S., Zhang, Y., Wang, T. H. \& Yang, S. Continuous dielectrophoretic bacterial separation and concentration from physiological media of high conductivity. $L a b$ Chip 11, 2893-2900 (2011).

2. Choi, S., Song, S., Choi, C. \& Park, J. K. Sheathless focusing of microbeads and blood cells based on hydrophoresis. Small 4, 634-641 (2008).

3. Shi, J., Mao, X., Ahmed, D., Colletti, A. \& Huang, T. J. Focusing microparticles in a microfluidic channel with standing surface acoustic waves (SSAW). Lab Chip 8, 221-223 (2008).

4. Liu, C., Stakenborg, T., Peeters, S. \& Lagae, L. Cell manipulation with magnetic particles toward microfluidic cytometry. J. Appl. Phys. 105 (2009).

5. Ai, Y., Mauroy, B., Sharma, A. \& Qian, S. Electrokinetic motion of a deformable particle: Dielectrophoretic effect. Electrophoresis 32, 2282-2291 (2011).

6. $\mathrm{Yu}, \mathrm{C} . \mathrm{H}$. et al. A three-dimensional dielectrophoretic particle focusing channel for microcytometry applications. J. Microelectromech. S. 14, 480-487 (2005).

7. Holmes, D., Morgan, H. \& Green, N. G. High throughput particle analysis: Combining dielectrophoretic particle focussing with confocal optical detection. Biosens. Bioelectron. 21, 1621-1630 (2006).

8. Cheng, I. F., Chang, H.-C., Hou, D. \& Chang, H.-C. An integrated dielectrophoretic chip for continuous bioparticle filtering, focusing, sorting, trapping, and detecting. Biomicrofluid. 1 (2007).

9. Demierre, N., Braschler, T., Muller, R. \& Renaud, P. Focusing and continuous separation of cells in a microfluidic device using lateral dielectrophoresis. Sensor. Actuat. B: Chem. 132, 388-396 (2008).

10. Cummings, E. B. \& Singh, A. K. Dielectrophoresis in microchips containing arrays of insulating posts: Theoretical and experimental results. Anal. Chem. 75, 4724-4731 (2003).

11. Thwar, P. K., Linderman, J. J. \& Burns, M. A. Electrodeless direct current dielectrophoresis using reconfigurable field-shaping oil barriers. Electrophoresis 28, 4572-4581 (2007).

12. Zhu, J. \& Xuan, X. Dielectrophoretic focusing of particles in a microchannel constriction using DC-biased AC flectric fields. Electrophoresis 30, 2668-2675 (2009).

13. Zhu, J., Tzeng, T.-R. J., Hu, G. \& Xuan, X. DC dielectrophoretic focusing of particles in a serpentine microchannel. Microfluid. Nanofluid. 7, 751-756 (2009).

14. Church, C. et al. Continuous particle separation in a serpentine microchannel via negative and positive dielectrophoretic focusing. J. Micromech.Microeng. 20 (2010).

15. Zhu, J. \& Xuan, X. Particle electrophoresis and dielectrophoresis in curved microchannels. J. Colloid Interface Sci. 340, 285-290 (2009).

16. Li, M., Li, S., Cao, W., Li, W. \& Wen, W. Continuous particle focusing in a waved microchannel using negative dc dielectrophoresis. J. Micromech.Microeng. 22, 095001 (2012)

17. Lee, G. B., Chang, C. C., Huang, S. B. \& Yang, R. J. The hydrodynamic focusing effect inside rectangular microchannels. J. Micromech.Microeng. 16, 1024-1032 (2006).

18. Chang, C. C., Huang, Z. X. \& Yang, R. J. Three-dimensional hydrodynamic focusing in two-layer polydimethylsiloxane (PDMS) microchannels. J. Micromech.Microeng. 17, 1479-1486 (2007).

19. Tsai, C. H., Hou, H. H. \& Fu, L. M. An optimal three-dimensional focusing technique for micro-flow cytometers. Microfluid. Nanofluid. 5, 827-836 (2008).

20. Kummrow, A. et al. Microfluidic structures for flow cytometric analysis of hydrodynamically focussed blood cells fabricated by ultraprecision micromachining. Lab Chip 9, 972-981 (2009).
21. Di Carlo, D. Inertial microfluidics. Lab Chip 9, 3038-3046 (2009).

22. Choi, S. \& Park, J.-K. Sheathless Hydrophoretic Particle Focusing in a Microchannel with Exponentially Increasing Obstacle Arrays. Anal. Chem. 80, 3035-3039 (2008).

23. Choi, S., Song, S., Choi, C. \& Park, J.-K. Hydrophoretic Sorting of Micrometer and Submicrometer Particles Using Anisotropic Microfluidic Obstacles. Anal. Chem. 81, 50-55 (2009).

24. Choi, S. \& Park, J.-K. Continuous hydrophoretic separation and sizing of microparticles using slanted obstacles in a microchannel. Lab Chip 7, 890-897 (2007).

25. Choi, S. \& Park, J.-K. Tuneable hydrophoretic separation using elastic deformation of poly(dimethylsiloxane). Lab Chip 9, 1962-1965 (2009).

26. Song, S. \& Choi, S. Design rules for size-based cell sorting and sheathless cell focusing by hydrophoresis. J. Chromatogr. A 1302, 191-196 (2013).

27. Xuan, X., Zhu, J. \& Church, C. Particle focusing in microfluidic devices. Microfluid. Nanofluid. 9, 1-16 (2010).

28. Markx, G. H., Talary, M. S. \& Pethig, R. Separation of viable and non-viable yeast using dielectrophoresis. J. Biotechnol. 32, 29-37 (1994).

29. Huang, Y., Wang, X. B., Becker, F. F. \& Gascoyne, P. R. Introducing dielectrophoresis as a new force field for field-flow fractionation. Biophys. J. 73 1118-1129 (1997).

30. Cheng, I. F., Froude, V. E., Zhu, Y. \& Chang, H. C. A continuous high-throughput bioparticle sorter based on 3D traveling-wave dielectrophoresis. Lab Chip 9, 3193-3201 (2009).

31. Regtmeier, J., Eichhorn, R., Viefhues, M., Bogunovic, L. \& Anselmetti, D. Electrodeless dielectrophoresis for bioanalysis: Theory, devices and applications. Electrophoresis 32, 2253-2273 (2011).

32. Huang, L. R., Cox, E. C., Austin, R. H. \& Sturm, J. C. Continuous particle separation through deterministic lateral displacement. Science 304, 987-990 (2004).

33. Davis, J. A. et al. Deterministic hydrodynamics: Taking blood apart. Proc. Natl. Acad. Sci. U. S. A. 103, 14779-14784 (2006)

34. Beech, J. P., Jonsson, P. \& Tegenfeldt, J. O. Tipping the balance of deterministic lateral displacement devices using dielectrophoresis. Lab Chip 9, 2698-2706 (2009).

35. Choi, S., Song, S., Choi, C. \& Park, J.-K. Continuous blood cell separation by hydrophoretic filtration. Lab Chip 7, 1532-1538 (2007).

36. Choi, S., Karp, J. M. \& Karnik, R. Cell sorting by deterministic cell rolling. Lab Chip 12, 1427-1430 (2012).

37. Jones, T. B. Electromechanics of Particles. 34-48, 110-138 (Cambridge Unviersity Press, 1995).

38. Pethig, R., Talary, M. S. \& Lee, R. S. Enhancing traveling-wave dielectrophoresis with signal superposition. IEEE Eng. Med. Biol. Mag. 22, 43-50 (2003).

39. Zhang, J., Li, M., Li, W. H. \& Alici, G. Inertial focusing in a straight channel with asymmetrical expansion-contraction cavity arrays using two secondary flows. J. Micromech.Microeng. 23, 085023 (2013).

40. Li, M. et al. A simple and cost-effective method for fabrication of integrated electronic-microfluidic devices using a laser-patterned PDMS layer. Microfluid. Nanofluid. 12, 751-760 (2012).

41. Sharp, K. V. \& Adrian, R. J. Transition from laminar to turbulent flow in liquid filled microtubes. Exp. Fluids 36, 741-747 (2004).

\section{Acknowledgments}

This work is supported by the University of Wollongong-China Scholarship Council joint scholarship. Special thanks are given to Ms. Aleta Pupovac for technical support with the MEL cell culture and immunofluorescence staining. 


\section{Author contributions}

S.Y., W.H.L., G.A., H.P.D. and R.S. designed the experiments, J.Z. and M.L. analysed data, S.Y. and W.H.L. co-wrote the manuscript. All authors contributed to manuscript presentation.

\section{Additional information}

Competing financial interests: The authors declare no competing financial interests.

How to cite this article: Yan, S. et al. On-chip high-throughput manipulation of particles in a dielectrophoresis-active hydrophoretic focuser. Sci. Rep. 4, 5060; DOI:10.1038/srep05060 (2014)
This work is licensed under a Creative Commons Attribution-NonCommercialShareAlike 3.0 Unported License. The images in this article are included in the article's Creative Commons license, unless indicated otherwise in the image credit; if the image is not included under the Creative Commons license, users will need to obtain permission from the license holder in order to reproduce the image. To view a copy of this license, visit http://creativecommons.org/licenses/by-nc-sa/3.0/ 